\title{
Room-temperature polarization switching and antiferromagnetic coupling in epitaxial $(\mathrm{Ga}, \mathrm{Fe})_{2} \mathrm{O}_{3} / \mathrm{SrRuO}_{3}$ heterostructures
}

Seol Hee Oh, Ji Hye Lee, Ran Hee Shin, Yooleemi Shin, Christian Meny, and William Jo

Citation: Appl. Phys. Lett. 106, 142902 (2015); doi: 10.1063/1.4917249

View online: https://doi.org/10.1063/1.4917249

View Table of Contents: http://aip.scitation.org/toc/apl/106/14

Published by the American Institute of Physics

\section{Articles you may be interested in}

Control of crystal-domain orientation in multiferroic $\mathrm{Ga}_{0.6} \mathrm{Fe}_{1.4} \mathrm{O}_{3}$ epitaxial thin films

Applied Physics Letters 110, 212905 (2017); 10.1063/1.4984211

Phase stability of multiferroic $\mathrm{GaFeO}_{3}$ up to $1368 \mathrm{~K}$ from in situ neutron diffraction

Journal of Applied Physics 113, 174102 (2013); 10.1063/1.4803679

Study of site-disorder in epitaxial magneto-electric $\mathrm{GaFeO}_{3}$ thin films

Applied Physics Letters 102, 212401 (2013); 10.1063/1.4807757

Epitaxial thin films of multiferroic $\mathrm{GaFeO}_{3}$ on conducting indium tin oxide (001) buffered yttrium-stabilized zirconia (001) by pulsed laser deposition

Applied Physics Letters 91, 202504 (2007); 10.1063/1.2813020

Origin of the different multiferroism in $\mathrm{BiFeO}_{3}$ and $\mathrm{GaFeO}_{3}$

Journal of Applied Physics 113, 063905 (2013); 10.1063/1.4791586

Reduced leakage currents and possible charge carriers tuning in $\mathrm{Mg}$-doped $\mathrm{Ga}_{0.6} \mathrm{Fe}_{1.4} \mathrm{O}_{3}$ thin films

Applied Physics Letters 100, 262904 (2012); 10.1063/1.4729872

\section{PHYSICS TODAY}

MANAGER'S GUIDE

Accelerate R\&D with

Multiphysics Simulation

\section{READ NOW}

PRESENTED BY

И $\subset$ OMSOL 


\title{
Room-temperature polarization switching and antiferromagnetic coupling in epitaxial $(\mathrm{Ga}, \mathrm{Fe})_{2} \mathrm{O}_{3} / \mathrm{SrRuO}_{3}$ heterostructures
}

\author{
Seol Hee Oh, ${ }^{1, \text { a) }}$ Ji Hye Lee, ${ }^{1, a)}$ Ran Hee Shin, ${ }^{1}$ Yooleemi Shin, ${ }^{2,3}$ Christian Meny, ${ }^{2}$ \\ and William Jo $\mathrm{J}^{1, \mathrm{~b})}$ \\ ${ }^{1}$ Department of Physics, Ewha Womans University, Seoul 120-750, South Korea \\ ${ }^{2}$ Institute of Physics and Chemistry of Materials of Strasbourg, UMR 7504, University of Strasbourg-CNRS, \\ Strasbourg 67043, France \\ ${ }^{3}$ CNRS-EWHA International Research Center, Ewha Womans University, Seoul 120-750, South Korea
}

(Received 4 February 2015; accepted 27 March 2015; published online 6 April 2015)

\begin{abstract}
Room-temperature reversible remnant polarization of gallium ferrite thin-films is reported as a multiferroic material with non-zero order parameters of polarization and magnetization. With the addition of $\mathrm{Fe}$ ions in $\mathrm{Ga}$ sites, $\mathrm{Ga}_{0.6} \mathrm{Fe}_{1.4} \mathrm{O}_{3}$ (GFO) thin films have been considered as potentially promising of multiferroicity. The $b$-axis oriented epitaxial GFO films were grown on $\mathrm{SrRuO}_{3}(111) /$ $\mathrm{SrTiO}_{3}(111)$. The six-fold symmetric in-plane epitaxy of the GFO films was confirmed using X-ray diffraction. The magnetic moment of the films was measured as a function of temperature and external magnetic field, which shows a room-temperature non-zero magnetization. Macroscopic and microscopic methods have been applied to demonstrate the polarization switching of the films. The remnant polarization is measured as $0.05 \mu \mathrm{C} / \mathrm{cm}^{2}$. Reduction of leaky behaviors of the GFO films owing to the conducting oxide of $\mathrm{SrRuO}_{3}$ will pave a way to take advantage of the roomtemperature non-zero multi-orders for future non-volatile memory device applications. (C) 2015 AIP Publishing LLC. [http://dx.doi.org/10.1063/1.4917249]
\end{abstract}

Multiferroics are materials that have two or more of the characteristics of ferroelectrics, ferromagnetics, and ferroelastics. ${ }^{1}$ In particular, magnetoelectric coupling in multiferroics has attracted much attention because the control of charges and spin by applied electric and magnetic fields could provide potential for applications in multiple controlled devices. ${ }^{2}$ While almost no true multiferroics with these two intrinsic ferroic orderings exist in nature, new compounds with such characteristics are being developed using advanced technology. The movement of metal ions to an asymmetrical location occurs due to the movement of charge density from a full $2 p$ state of oxygen ions to an empty $d$-state of transition metal in the perovskite type oxide. ${ }^{3}$ In contrast, the d-state must be partially filled to produce a magnetic spin moment in the transition metal. Therefore, the condition of the empty $d$-state in ferroelectric materials clashes with the need for $d$-electrons to have a spin moment. For that reason, multiferroics with both ferroelectric and ferromagnetic properties are interesting with some exotic physical phenomena. ${ }^{4-8}$

$\mathrm{Ga}_{0.6} \mathrm{Fe}_{1.4} \mathrm{O}_{3}$ (GFO) has been reported as a promising multiferroic material for magnetoelectric devices because it has non-zero remnant magnetization at room temperature. $^{9-13}$ Fabrication of the GFO thin films on indium tin oxide (ITO)/yttrium-stabilized zirconia (YSZ) and $\mathrm{Pt} / \mathrm{Ti} / \mathrm{SiO}_{2} /$ Si substrates has been reported. ${ }^{12,14}$ While an early report attributed asymmetrically placed Ga1 ions within the unit cell responsible for observed piezoelectric response of GFO. ${ }^{15}$ However, recent first-principles calculations showed that the electric polarization takes into account the

\footnotetext{
${ }^{\text {a) }}$ S. H. Oh and J. H. Lee contributed equally to this work.

${ }^{b)}$ Author to whom correspondence should be addressed. Electronic mail: wmjo@ewha.ac.kr
}

displacements of $\mathrm{Fe}$ ions in oxygen octahedrons. ${ }^{16}$ Furthermore, a hint towards possible ferroelectric switching was reported by Stoeffler, ${ }^{10}$ by considering GFO structure from the non-centrosymmetric position (space group: Pna2 $2_{1}$ ) to the centrosymmetric positions (space group: Pnna). Thus, it is likely that our GFO films exhibit a reversible polarization under a certain condition. However, leaky polarizationelectric field hysteresis curves were reported in the GFO due to the large leakage current. ${ }^{17}$ The main origin of the large leakage current is electron hopping between $\mathrm{Fe}^{2+}$ and $\mathrm{Fe}^{3+}$ sites. ${ }^{18}$ Therefore, suitable substrates have been introduced that show less leaky behavior of the thin-films. In addition, to determine the interaction property between two ferrimagnetic/ferromagnetic layers in spin-spin interaction, charge ordering, and orbital ordering, an alternative matching for electrode to GFO is needed. $\mathrm{SrRuO}_{3}$ (SRO) is a good candidate for electrodes as it is not only a good ferromagnetic material but also has excellent crystallographic matching to GFO, despite the somewhat large mismatch $(\sim 9 \%)$ compared to that of between ITO and GFO $(\sim 0.4 \%)$. SRO has been also demonstrated to exhibit unique anisotropic properties that arise from the dependence of their magnetic and resistive behaviors on various crystallographic directions in their structure. ${ }^{19}$ Despite the relatively intense research on magnetic interaction in superlattices or multilayers films, ${ }^{20}$ no report has been found on this configuration of $\mathrm{Fe}$ and $\mathrm{Ru}$ in GFO/SRO. In this paper, we present room-temperature remnant polarization switching of the GFO epitaxial thin films on SRO as well as their magnetic properties over a wide range of temperature.

The heterostructures of GFO/SRO were grown on (111) oriented $\mathrm{SrTiO}_{3}$ (STO) substrates by pulsed laser deposition using a $\mathrm{KrF}$ excimer laser $(\lambda=248 \mathrm{~nm})$ with a $5 \mathrm{~Hz}$ 
repetition rate and a fluence of $2 \mathrm{~J} / \mathrm{cm}^{2}$. The different materials could be deposited in-situ using a multi-target system to fabricate multilayered films. First, a $200 \mathrm{~nm}$ thick SRO conducting layer was deposited at $650{ }^{\circ} \mathrm{C}$ on a STO (111) substrate with 200 mTorr oxygen partial pressure and a $200 \mathrm{~nm}$ thick GFO layer was then deposited at $750^{\circ} \mathrm{C}$ with 250 mTorr oxygen partial pressure. The samples then underwent a cooling process at room temperature at gas deposition pressure.

The entire crystallographic structure of each sample was characterized by X-ray diffraction $\theta-2 \theta$ scans, rocking curve, and $\varphi$-scan measurement. The magnetic properties were studied using a X-ray superconducting quantum interference device magnetometer (Quantum Design). The current versus applied electric field relation was investigated in a probestation system using semiconductor parameter analysis (HP4145B). Investigation of the switchable polarization of GFO is a very important key factor because it can demonstrate a natural multiferroic property. Therefore, switchable polarizations using a scanning probe microscope technique and a TF analyzer (Radiant 6000) have also been studied.

A preliminary structure analysis was performed for GFO/SRO/STO thin films using $\theta-2 \theta$ scans as shown in Fig. 1(a). The pure $b$-axis growth of GFO is clearly shown. The GFO crystalline quality is kept high as shown by the rocking curve FWHM of $0.71^{\circ}$ observed for the GFO (040) peak in the inset of Fig. 1(a). Phi-scans of the GFO $\{042\}$ and SRO $\{110\}$ planes were carried out. They present the $60^{\circ}$ periodicity for both GFO and SRO peaks in Fig. 1(b). The GFO crystallites present three variants having in-plane orientations every $60^{\circ}$. Compared to ITO electrode, ${ }^{12}$ we can expect the reduction of the GFO in-plane variants using the SRO (111) electrode, in which six variants are shown. Although lattice-mismatch of the GFO with SRO electrode $(\sim 9 \%)$ is larger than ITO electrode $(\sim 0.4 \%),{ }^{17}$ it is remarkable that the films show highly crystalline quality of epitaxy. Therefore, the epitaxial relations could be determined: $\operatorname{GFO}(010) / / \operatorname{SRO}(111) / / \mathrm{STO}(111)$ for the in-plane and GFO[100]//SRO[110]//SRO[110] for the out-of-plane.

Magnetization measurements were performed on $3 \times 3 \mathrm{~mm}^{2}$ samples of GFO/SRO/STO thin-films, in the two perpendicular in-plane and out-of-plane directions of the thin-films. The applied magnetic field was 100 Oe along the film. The temperature dependent zero field cooled (ZFC) and field cooled (FC) magnetization data $(M-T)$ are shown in Figs. 2(a) and 2(c) with different magnetic field directions. At $T<T_{\mathrm{c}}$, the ZFC and FC branches show strong divergence due to freezing of the moments of SRO by the GFO in the ZFC state. Unique behavior is observed in the magnetic field dependence of magnetization in the thin-films along the in-plane applied magnetic field shown in Fig. 2(b). Especially near $H_{\mathrm{c}}$, two plateaus are shown corresponding to the $180^{\circ}$ magnetization reversal of the sample, in which the spin arrangement flips from parallel to antiparallel due to the indirect exchange interaction of the ferromagnetic ordering of the magnetic moments between the interfaces of the two thin-films. ${ }^{21}$ When the magnetic field was applied inplane of the thin-films, from 160 to $360 \mathrm{~K}$, the difference of zero field cooling and the magnetization of the field cooling increased more than that in out-of plane as shown in
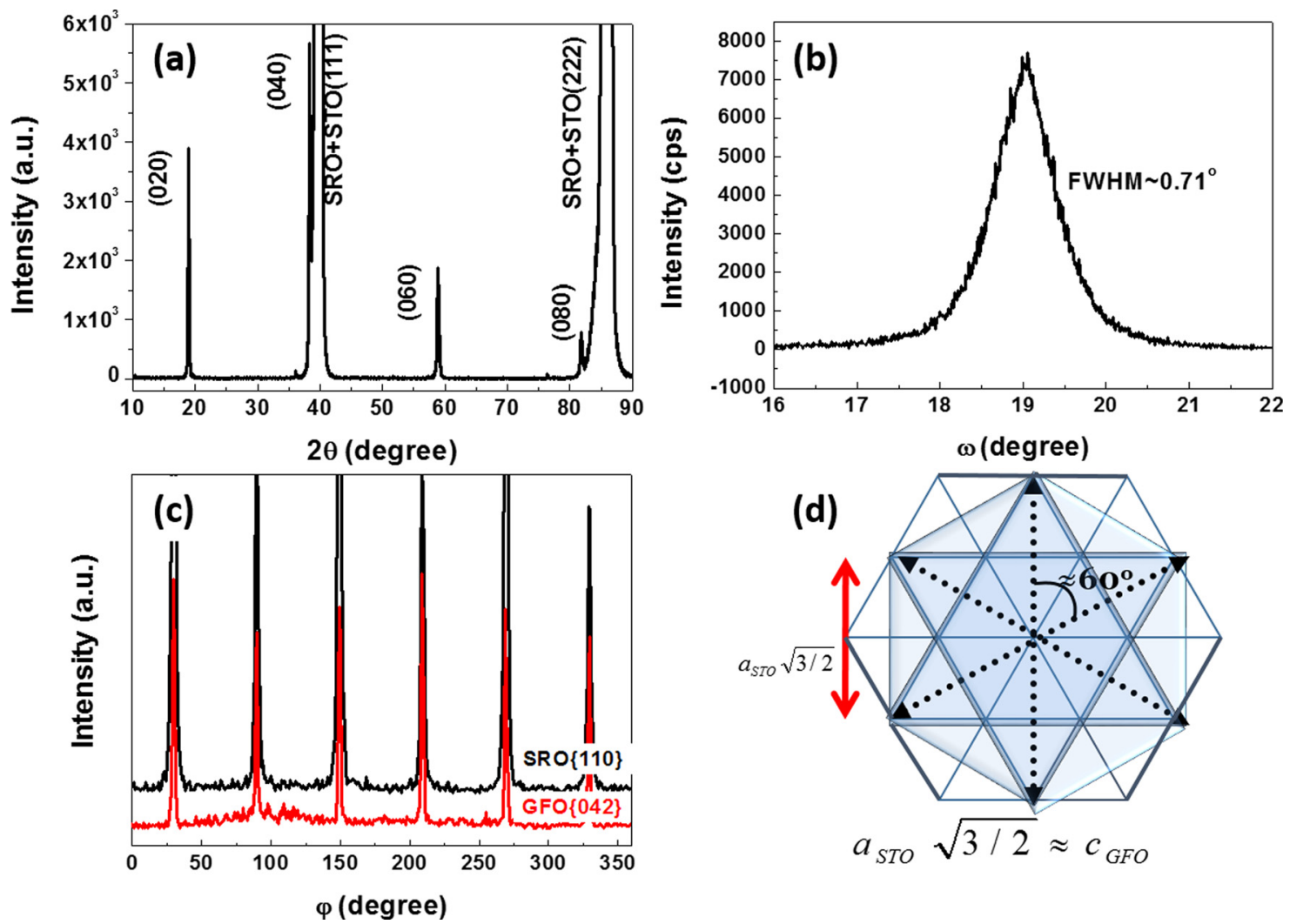

FIG. 1. X-ray diffraction patterns of a GFO/SRO/STO thin-film. (a) A $\theta-2 \theta$ scan of the GFO film, (b) a rocking curve of the GFO (040) peak, (c) $\varphi$-scans of the GFO (042) and SRO (110) planes showing a six-fold symmetry for GFO and SRO. (d) The resultant in-plane possible orientations of GFO (0k0) cells on the STO (111) surface. 

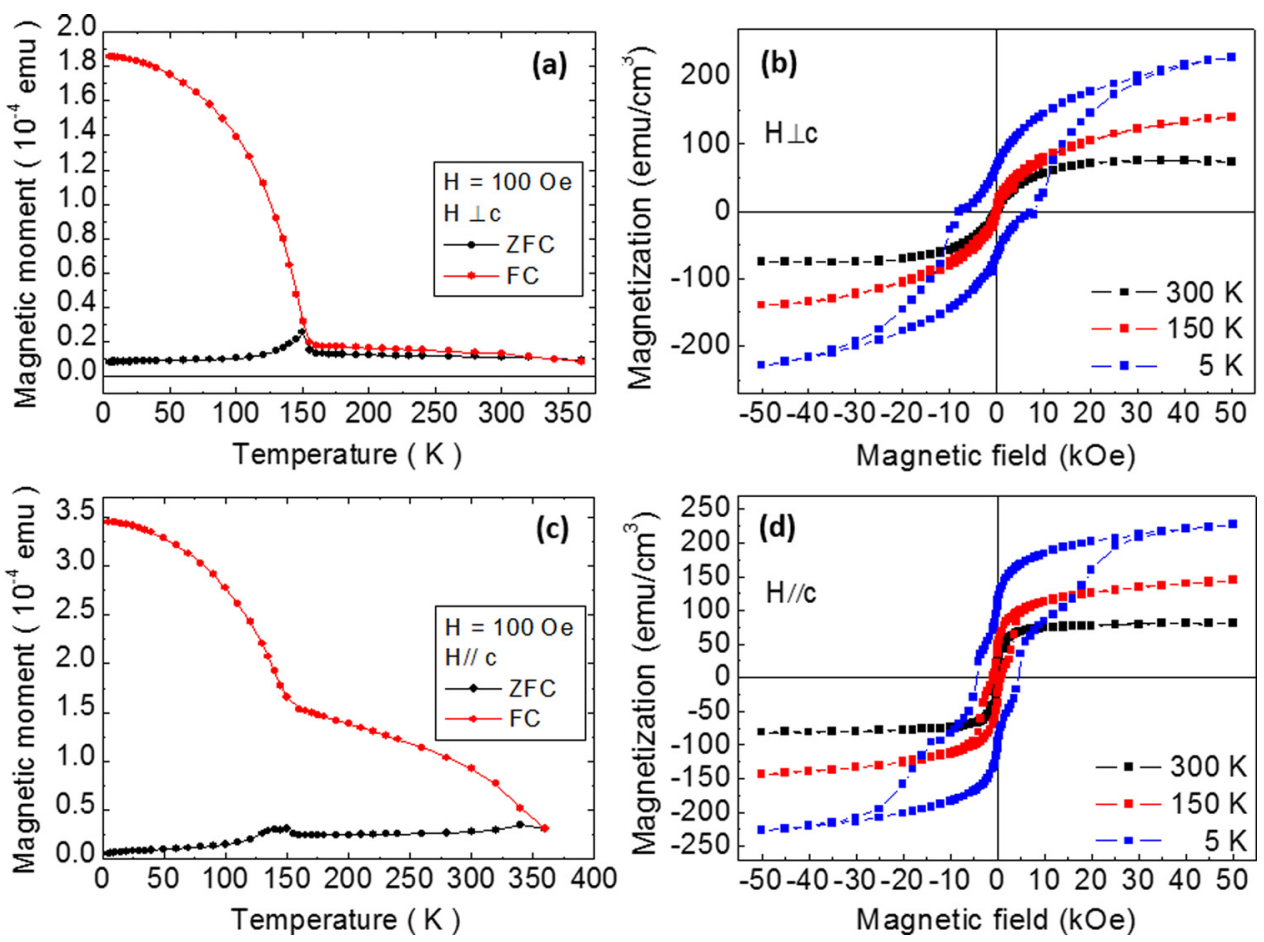

FIG. 2. (a) Temperature dependent out-of-plane magnetization of the GFO/SRO/STO thin-film under 100 Oe. (b) Magnetization vs. magnetic field of the GFO/SRO/STO thin-film. (c) Temperature dependent in-plane magnetization of the GFO/SRO/STO thin-film under 100 Oe. (d) Magnetization vs magnetic field of the GFO/SRO/STO thin-film.

Fig. 2(c). The variation of hysteresis loop with temperature shown in Fig. 2(d) reflects the change of the interlayer coupling between GFO and SRO and the step gradually disappears with increasing temperatures due to the increase of interlayer coupling. The antiferromagnetic coupling may occur due to $\mathrm{Ru}-\mathrm{O}-\mathrm{Fe}$ bond at interface and is enhanced by replacing Ga with $\mathrm{Fe}^{22-24}$ At present, it is not clear whether a magnetoelectric interaction involves with the materials. But, it is very intriguing to study the effects of the stacking orders between GFO and SRO layers on magnetic and electric properties of the heterostructures.

Fig. 3 shows the current versus applied electric field graphs of the GFO/SRO/STO and GFO/ITO/YSZ thin-films. In order to measure their electrical properties, the Pt is used as a top electrode with a dot shape deposited using e-beam

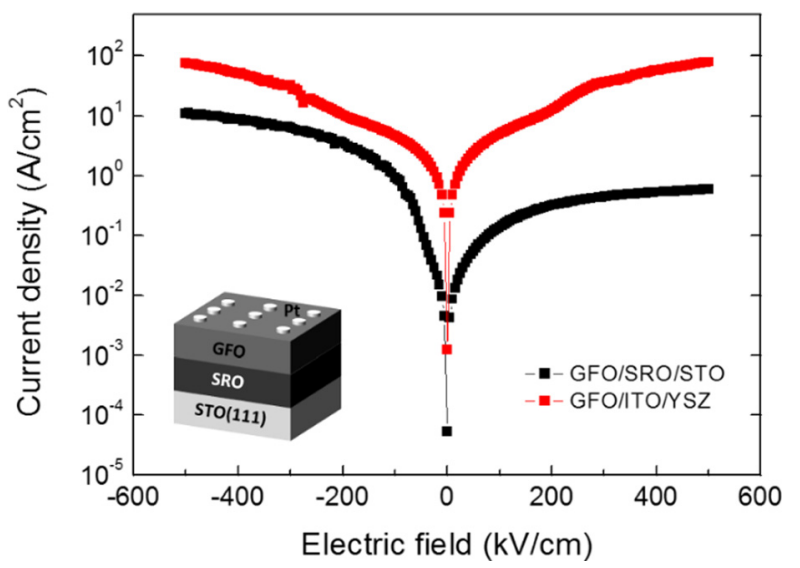

FIG. 3. Current density vs. electric field curves of the GFO/SRO/STO film (in this work) and the GFO/ITO/YSZ film. ${ }^{12} \mathrm{Pt}$ was used as top electrodes for the transport measurement. evaporation technique. This clearly shows the influence of the bottom electrode on the measured leakage current. The GFO thin-films deposited on the SRO/STO electrodes exhibiting excellent crystallization showed lower leakage current density compared to the GFO thin films deposited on ITO/ YSZ. In the GFO/SRO/STO heterostructure, the number of GFO in-plane variants is reduced from six to three. While the ITO (001) surface is cubic, the hexagonal symmetry exhibited by the SRO (111) surface results in the reduction of the matching possibilities. High leakage is responsible for artifacts in the measurement of polarization-electric field $(P-E)$ loops resulting in cigar-shaped loops that are misinterpreted as ferroelectric loops. ${ }^{25}$ In addition to, an asymmetric electric characteristic has been commonly observed at ferroelectric or multiferroic materials, in which a diode-like behavior was reported by Choi et al. ${ }^{26}$ However, the asymmetric electric current-voltage behavior can be changed by interface between the bottom electrodes and the GFO film, which is shown in the results of the GFO/ITO/YSZ thin film.

The important aspect of this material is to investigate polarization because it has been known to be pyroelectric, which means that polarization is spontaneous but non switchable. However, to use this material in multi-bit storage using multiferroic characteristics, switchable polarization occurs with applied electric field. However, the reports on the investigation of ferroelectric characteristics of this material both in single crystal and thin-films system showed leaky dielectric behaviors of $P-E$ loops ${ }^{17,27}$ Fig. 4 shows the topography, amplitude, and phase information of the GFO/ SRO/STO thin-films. We can see the distinct contrasts in the PFM images that indicated the different domain configurations in which the direction of polarization is opposite on each region. As-grown polarization states, shown in 

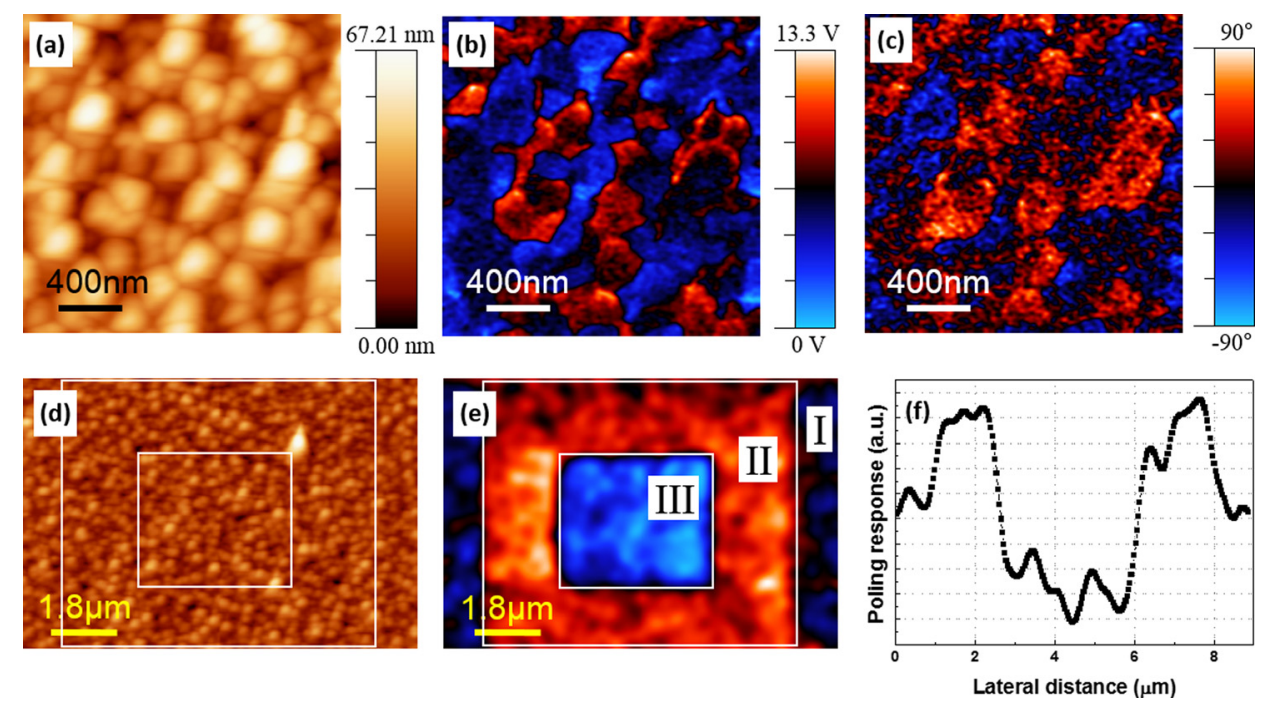

FIG. 4. Piezoelectric microscopic results of the GFO/SRO/STO film. (a) Topography, (b) the amplitude image of the PFM scanning, and (c) the phase image of the PFM scanning. (d) A topographic image for the poling process. (e) A poling image which is corresponding to the topography in Fig. 4(e). (f) A cross-sectional line profile of the double poling area in the GFO/SRO/STO thin-film. The profile clearly shows the switching characteristics of the GFO layer. The $+10 \mathrm{~V}$ and $-10 \mathrm{~V}$ poled regions show similar poling magnitude of the PFM response.
Figs. 4(b) and 4(c), exhibit the upward and downward polarization in the GFO films. In Fig. 4(e), the region I is an asgrown state, the region II is the single-poling state with a bias $-10 \mathrm{~V}$, and the region III is the double poling state with a bias $+10 \mathrm{~V}$ following a $-10 \mathrm{~V}$. According to our PFM measurement, the poled region is bigger than the size of single grains of the GFO thin film, which means that polarizations at grain boundaries and grain interiors were switched by the applied electric field, as shown in Figs. 4(d) and 4(e). The microscopic hysteresis loop in Fig. 5(b) is closely related to the poling response in the PFM measurement which is clearly shown in Fig. 4(e). Therefore, insulating grain boundaries may not the source of the hysteretic behaviors. ${ }^{28}$ The cross sectional line profile of this line was then obtained as shown in Fig. 4(f). The PFM image indicates that the perpendicular component of polarization can be switched between the two stable states. The double poled PFM image has been reported in other ferroelectric materials like $\mathrm{PbMg}_{1 / 3} \mathrm{Nb}_{2 / 3} \mathrm{O}_{3}-\mathrm{PbTiO}_{3}$ thin films or $\mathrm{Nd}$-substituted $\mathrm{Bi}_{4} \mathrm{Ti}_{3} \mathrm{O}_{12}$ thin films by our group. ${ }^{29,30}$ From the doublepoling scheme measurement, it is confirmed that the domains in the GFO films are switchable and have similar magnitude of polarization states.

Furthermore, the macroscopic $P-V$ curves were measured at room temperature by changing the frequency and amplitude of the applied AC voltage. In Fig. 5(a), the frequency dependent $P-V$ curves observed show saturated hysteretic behavior over the whole frequency range. The switching behavior reveals that contribution of DC leakage current cannot screen the polarization reversal in our films. The $P-V$ curve measured at $f=8 \mathrm{kHz}$ frequency with applied electric field from $-5 \mathrm{~V}$ to $+5 \mathrm{~V}$ represents that the obtained remnant polarization value is about $0.05 \mu \mathrm{C} / \mathrm{cm}^{2}$. However, this value is very small compared to the reported results in multiferroic materials. The predicted polarization values along the $b$-axis of GFO by theoretical calculations have been reported to be $59 \mu \mathrm{C} / \mathrm{cm}^{2}$ or $25 \mu \mathrm{C} / \mathrm{cm}^{2}$, respectively. ${ }^{9,10}$ Typically, it is hard to match with expectation value because calculation would be performed for the case of full switching of polarization. Thomasson et al. reported experimentally remnant polarization of $0.2 \mu \mathrm{C} / \mathrm{cm}^{2}$ at $2.5 \mathrm{kHz}$ for $\mathrm{Mg}$-doped $\mathrm{GFO}$ films. ${ }^{31}$ Among some other multiferroic materials, $\mathrm{YMnO}_{3}$ has been considered as ferroelectric material with small remnant polarization $2 \mu \mathrm{C} / \mathrm{cm}^{2}$, which is because long-range dipole-dipole interactions and oxygen rotations both cooperate to drive the system towards the stable ferroelectric state. $^{32,33}$ Therefore, it needed an extra layer which is magnetically coupled with BFO layers. This would make the application of devices more complicated. Furthermore, the
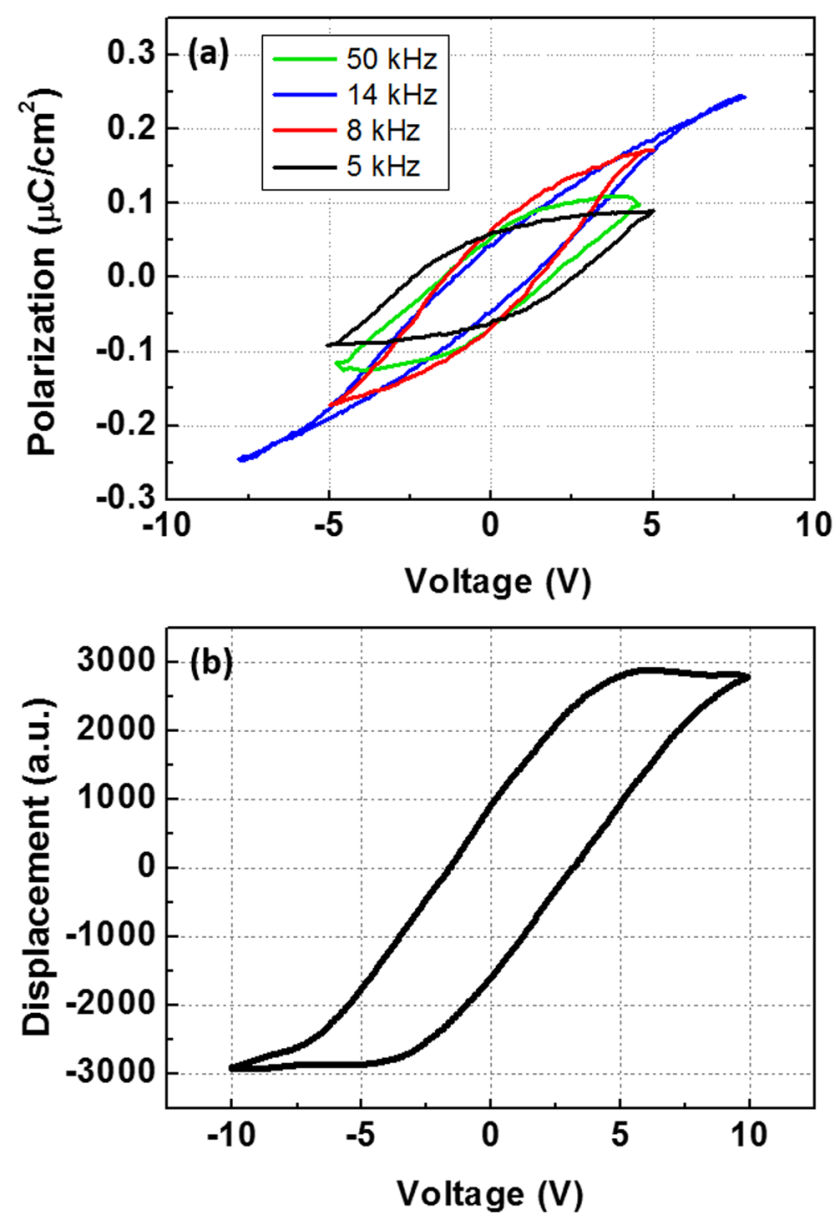

FIG. 5. Polarization vs. voltage $(P-V)$ loops of the GFO/SRO/STO thin-film. (Film thickness $=200 \mathrm{~nm}$ ) (a) Macroscopic $P$ - $V$ curves with Pt tip electrodes with different frequencies measured at room temperature. (b) Microscopic measurement with a Pt-coated tip. 
GFO films deposited on SRO/STO substrates show saturated polarization behavior in its $P-E$ loops measured by piezoresponse force microscopy (PFM) at room temperature shown in Fig. 5(b). These results corresponded with the macroscopic results measured at room temperature shown in Fig. 5(a). The GFO/SRO/STO represents well-saturated hysteretic behavior in local measurement. Although this measurement is significantly slower than that in macroscopic results, in which time interval and responses of polarization are quite fast, it suggests that this material has switchable polarization in a thin-films system. The fundamental question has been raised of the possible mechanisms for multiferroic behavior that can exist in this material. Here, we consider multiferroicity that is driven by different forms of charge ordering.

In conclusion, room-temperature polarization switching of GFO thin films is observed with relatively small remnant polarization values. Highly $b$-axis oriented GFO thin films with six-fold in-plane symmetry were obtained on SRO/STO (111). With the heterostructure, owing to significant reduction of leakage currents, macroscopic and microscopic revealing of room-temperature polarization switching is confirmed. Furthermore, an interesting coupling behavior between GFO and SRO is examined.

This research was supported by a National Research Foundation of Korea (NRF) grant funded by the Korean Government (MSIP) (No. 2014-004070) and one of the authors (SHO) was supported by an NRF grant funded by the Korean Government (NRF-2013-Fostering Core Leaders of the Future Basic Science Program).

${ }^{1}$ N. A. Spaldin and M. Fiebig, Science 309, 391 (2005).

${ }^{2}$ M. Bibes and A. Barthelemy, Nat. Mater. 7, 425 (2008).

${ }^{3}$ R. E. Cohen and H. Karkauer, Ferroelectrics 136, 65 (1992).

${ }^{4}$ S. Ju, T.-Y. Cai, G.-Y. Guo, and Z.-Y. Li, Phys. Rev. B 75, 064419 (2007).

${ }^{5}$ T. Arima, J. Phys. Soc. Jpn. 80, 052001 (2011).

${ }^{6}$ R. Ramesh and N. A. Spaldin, Nat. Mater. 6, 21 (2007).

${ }^{7}$ S. M. Wu, S. A. Cybart, P. Yu, M. D. Rossell, J. X. Zhang, R. Ramesh, and R. C. Dynes, Nat. Mater. 9, 756 (2010).
${ }^{8}$ S. H. Baek, H. W. Jang, C. M. Folkman, Y. L. Li, B. Winchester, J. X. Zhang, Q. He, Y. H. Chu, C. T. Nelson, M. S. Rzchowski, X. Q. Pan, R. Ramesh, L. Q. Chen, and C. B. Eom, Nat. Mater. 9, 309 (2010).

${ }^{9}$ A. Roy, S. Mukherjee, R. Gupta, S. Auluck, R. Prasad, and A. Garg, J. Phys.: Condens. Matter 23, 325902 (2011).

${ }^{10}$ D. Stoeffler, J. Phys.: Condens. Matter 24, 185502 (2012).

${ }^{11}$ G. T. Rado, Phys. Rev. Lett. 13, 335 (1964).

${ }^{12}$ M. Trassin, N. Viart, G. Versini, S. Barre, G. Pourroy, J. H. Lee, W. Jo, K. Dumesnil, C. Dufour, and S. Robert, J. Mater. Chem. 19, 8876 (2009).

${ }^{13}$ C. H. Nowlin and R. V. Jones, J. Appl. Phys. 34, 1262 (1963).

${ }^{14}$ M. Trassin, N. Viart, G. Versini, J.-L. Loison, J.-P. Vola, G. Schmerber, O. Cregut, S. Barre, G. Pourroy, J. H. Lee, W. Jo, and C. Meny, Appl. Phys. Lett. 91, 202504 (2007).

${ }^{15}$ S. C. Abrahams and J. M. Reddy, Phys. Rev. Lett. 13, 688 (1964).

${ }^{16}$ T. Arima, D. Higashiyama, Y. Kaneko, J. P. He, T. Goto, S. Miyasaka, T. Kumura, K. Oikawa, T. Kamiyama, R. Kumai, and Y. Tokura, Phys. Rev. B 70, 064426 (2004).

${ }^{17}$ Z. H. Sun, Y. L. Zhou, S. Y. Dai, L. Z. Cao, and Z. H. Chen, Appl. Phys. A 91, 97 (2008).

${ }^{18}$ C. Lefèvre, R. H. Shin, J. H. Lee, S. H. Oh, F. Roulland, A. Thomasson, E. Autissier, C. Mény, W. Jo, and N. Viart, Appl. Phys. Lett. 100, 262904 (2012).

${ }^{19}$ J. F. Scott, Ferroelectric Memories (Springer, Berlin, Germany, 2000).

${ }^{20}$ A. Gruverman, O. Auciello, and H. Tokumoto, Annu. Rev. Mater. Sci. 28, 101 (1998).

${ }^{21}$ D. I. Sementsov and A. M. Shutyi, Crystallogr. Rep. 49, 1042 (2004).

${ }^{22}$ H.-C. Wu, O. N. Mryasov, M. Abid, K. Radican, and I. V. Shvets, Sci. Rep. 3, 1830 (2013).

${ }^{23}$ I. Knittel, J. Wei, Y. Zhou, S. K. Arora, I. V. Shvets, M. Luysberg, and U. Hartmann, Phys. Rev. B 74, 132406 (2006).

${ }^{24}$ W. Kuch, L. I. Chelaru, F. Offi, J. Wang, M. Kotsugi, and J. Kirschner, Nat. Mater. 5, 128 (2006).

${ }^{25}$ J. F. Scott, J. Phys.: Condens. Matter 20, 021001 (2008).

${ }^{26}$ T. Choi, S. Lee, Y. J. Choi, V. Kiryukhin, and S.-W. Cheong, Science 324, 63 (2009).

${ }^{27}$ V. B. Naik and R. Mahendiran, J. Appl. Phys. 106, 123910 (2009).

${ }^{28}$ J. H. Lee, M. R. Choi, Y. J. Oh, and W. Jo, Appl. Phys. Lett. 91, 072906 (2007).

${ }^{29}$ J. H. Lee, R. H. Shin, and W. Jo, Phys. Rev. B 84, 094112 (2011).

${ }^{30}$ A. Thomasson, S. Cherifi, C. Lefèvre, F. Roulland, B. Gautier, D. Albertini, C. Meny, and N. Viart, J. Appl. Phys. 113, 214101 (2013).

${ }^{31}$ J. Wang, W. Shu, H. Fang, and M. Kamlah, Smart Mater. Struct. 23, 095016 (2014).

${ }^{32}$ A. Posadas, J.-B. Yau, C. H. Ahn, J. Han, S. Gariglio, K. Johnston, K. M. Rabe, and J. B. Neaton, Appl. Phys. Lett. 87, 171915 (2005).

${ }^{33}$ B. B. V. Aken, T. T. M. Palstra, A. Filippetti, and N. A. Spaldin, Nat. Mater. 3, 164 (2004). 